\title{
Co-infection of Candida albicans and Trichomonas vaginalis among Pregnant Women in Tertiary Care Hospital
}

\author{
Surbhi Mishra and Arti Jain*
}

\begin{abstract}
Department of Microbiology, People's College of Medical Sciences \& Research Center, Bhanpur Road, Bhopal -462037, Madhya Pradesh, India
\end{abstract}

*Corresponding author

\section{A B S T R A C T}

\section{Ke ywords \\ Trichomonas vaginalis, Candida albicans and Pregnant women \\ Article Info \\ Accepted: \\ 20 August 2020 \\ Available Online: \\ 10 September 2020}

\begin{abstract}
Vaginal infections are common among women of reproductive age. The purpose of this study was Co-infection of Candida albicans and Trichomonas vaginalis among pregnant woman in Tertiary care hospital. The prospective Analytical study was conducted in the Department of Microbiology, attached to a Tertiary Care Hospital in Central India after obtaining ethical clearance over a time period of one year. Out of these 320 samples, 200 sample were randomly selected (by Chit system) in our study. Out of 200 samples 46 (23\%) samples were culture positive. VVC, Trichomoniasis and other vaginal infection can resolve within a short period of time, if not treated timely then, it may enhances the growth of co-infection and it is a major cause of morbidity in pregnant women in developing countries like India. Present study highlights Rapid test should be mandatory routinely done for all pregnant women or in patients suffering from vaginal discharge and thus, help in early diagnosis and prompt treatment of patients.
\end{abstract}

\section{Introduction}

Vaginal infections are common infection in women. They can be caused by sexually transmitted microorganisms or by some disorder resulting in the proliferation of endogenous microbials ${ }^{1}$. Among the more frequent microorganisms are the protozoan Trichomonas vaginalis (T. vaginalis) $)^{2}$ and Candida albicans (C. albicans) responsible for vaginitis specially in women of childbearing age ${ }^{3}$.

These organisms may occur as single infection or co-infections. Mucosal candidiasis, especially vulvovaginal candidiasis (VVC), is one of the most population suffers at least one episode during their lives ${ }^{4}$ Pregnancy, diabetes mellitus and antibiotic treatment are the most common predisposing factors ${ }^{5,6}$ Some studies have shown that vulvovaginitis has increased in the past three decades due to antifungal resistance in the Candida species and a change in women's health quality. ${ }^{7,89}$

Candidiasis is associated with vaginal discharge and pruritis. The discharge appears to be like curded milk or white discharge, soreness, dyspareunia, irritation and itching and deep erythema of vulva and vagina is often seen. The incidence of the infection is 
almost double in pregnant women particularly in the third trimester, compare to nonpregnant women. ${ }^{10}$

Vaginitis due to Trichomonas vaginalis clinically manifests with symptoms of vaginal itching, odor, purulent, foul-smelling and vaginal discharge, which is associated with pruritis, dysuria and dyspareunia. ${ }^{11}$

In various clinical settings, culture is not available for detection of $T$. vaginalis and it requires additional resources. Wet mount is the most widely used method for the detection of $T$. vaginalis in clinically suspected cases of vulvovaginitis. ${ }^{12}$

Although, wet mount examination is most cost-effective diagnosis test, but it has low sensitivity resulting in under diagnosis of the diseases. Therefore, to overcome this problem, various staining techniques like Giemsa and Acridine orange can be used along with wet mount examination for diagnosis of $T$. vaginalis infection.

This study was emphasizing the importance of screening and treating infected pregnant women to prevent these unwanted infections. Therefore, this study was conducted to determine the Co-infection of Candida albicans and Trichomonas vaginal is among pregnant woman attending antenatal clinic in Tertiary care hospital.

\section{Materials and Methods}

This Prospective analytical study was carried out in the Department of Microbiology, in college associated with Tertiary care hospital, in central India over a time period of one year after obtaining Ethics Clearance

All pregnant women presenting with signs and symptoms of vaginitis reporting in OBGY were enrolled.

\section{Collection of Samples}

A total 200 vaginal samples were collected by clinicians in suspected cases of vulvovaginitis after written informed consent.

The clinicians were obtained three vaginal samples/swabs [Hi-media laboratories Pvt. Limited, Mumbai]

First swab was used for detection of motile Trichomonas \& budding yeast cells by wetmount microscopy \& second swab was used to prepare smear for Giemsa staining, Gram staining \& Acridine orange staining and third swab was used for culture onto Blood agar and Sabouraud's dextrose agar for detection the growth of candida from each patient by swabbing the vaginal vault with the help of vaginal speculum and then send to laboratory within 10 min of collection.

\section{Procedure for sampling}

Three vaginal samples were collected by clinician. The samples were transported immediately to the microbiology laboratory within 10 minutes of collection.

After the receiving of sample in the laboratory, will be processed as follows-

Macroscopic Examination-each sample was examined for color, appearance \& odor.

$\mathrm{I}^{\text {st }}$ swab - was placed in screw capped sterile plastic tube containing $0.5 \mathrm{ml}$ of $0.9 \%$ saline and used immediately for detection of motile Trichomonas and budding yeast cells to rule out the Candida by wet-mount microscopy.

The Trichomonads were identified by their size $(10-20 \mu \mathrm{m})$, round or oval shape, and characteristic twitching motility and Candida were identified by their size $(3-5 \mu \mathrm{m})$, ovoid or spherical budding yeast cells by wet mount examination. 
II swab was used to prepare smear for Giemsa staining, Acridine orange staining for detection of Trichomonas vaginalis and Gram staining detection of candida morphology (Gram positive budding yeast cells) (Procedure as per manufactures).

\section{Giemsa Staining}

The prepared smear was scanned for Trichomonas vaginalis at $10 \mathrm{X}$ magnification and then at a magnification of 40xAt least 30 fields were examined before a negative finding was recorded.

Both the internal and external structures of the organisms were clearly visualized. The former stained dark blue with a red nucleus and the latter was sharply being outlined, showing clearly the flagella and the undulating membrane.

\section{Acridine Orange Staining}

The prepared smear was fixed with methanol for 2-3 min and allow to dry it. It was then stained with Acridine Orange Staining as per as manufactures instructions. After staining, the slide was examined under a fluorescence microscope (with a 470-490 nm filter) at a magnification of $40 \mathrm{Xf}^{13}$ Epithelial cells fluoresced light green with a bright green nucleus. The nuclei of leucocytes (pus cells) fluoresced bright green and bacteria stained bright red color. Trophozoites of $T$. vaginalis were seen as characteristic brick red color with a yellowish green nucleus.

\section{Gram staining}

The prepared smear was stained by Gram staining as per as manufactures instructions. After staining the slide were examined by light microscope for detection of Candida morphology (Gram positive budding yeast cells)
III swab was used for culture onto Blood agar and Seaboard's dextrose agar for detection the growth of candida. Procedure was as follows:

The colonies on BA \& SDA were further be identified and species identification by CHROM agar then antifungal susceptibility was performed by standard procedure.

\section{Results and Discussion}

A total of 200 vaginal swabs were collected from patient of reproductive age group (15-45 years) after Institutional Ethics committee clearance.

A total of 3158 pregnant women were seen at the OBGY department of a Tertiary care centre, over a period of 12 months from $\left(1^{\text {st }}\right.$ May 2017 to $31^{\text {st }}$ May 2018). Out of these 320 women, (10\%) presented with vaginal discharge and were sent for high vaginal swabs microscopy, culture and sensitivity. Out of these 320 samples, 200 sample were randomly selected (by Chit system) in our study. Out of 200 samples 46 (23\%) samples were culture positive.

The Age wise distribution of patients having vaginal discharge among pregnant women were collected is as given in Table 1 .

\section{Frequency of Candidiasis}

Out of 200 samples, $42(21 \%)$ cases of vaginal infection were positive for candidiasis among these women.

Maximum number of candidiasis were positive in the age group of 20 to 30 years 21 $(10.5 \%)$, followed by age group 30 to 40 years $17(8.5 \%)$. There were $4(2.5 \%)$ in $40-45$ years

The observation by Direct wet mount, Gram staining, Culture results of Candidiasis among pregnant women were as shown in Table 2 . 
Out of 42 positive candidiasis, Candida albicans were maximum 36 then non-albicans that was only 6 .

Detection of different spp of isolated Candida by culture on CHROMagar

Out of the 42 isolated Candiada spp in pregnant women, maximum no. of candida albicans were positive (36), followed by $\mathrm{C}$. dublinansis (3), C. krusei(2) and C. glabrata(1).

Percentage of Candidiasis in different trimester among pregnant women were shown in Figure 1. Anti- fungal sensitivity pattern of the predominant Candida isolated from vaginal infection among pregnant women were shown in Table 3.

\section{Frequency of Trichomoniasis}

Frequency of Trichomoniasis in various age groups among pregnant women shown in Table 4.

Observation by Direct wet mount, Gram staining, Culture results of Trichomonissis among pregnant women shown in Table 5.

Percentage of Trichomoniasis in different trimester among pregnant women shown in Figure 2.

\section{Frequency of co-infection}

Out of 200 samples, $46(23 \%)$ cases of vaginal infection were positive among these women. of $42(21 \%)$ were candida infection followed by 04 (2\%) trichomonas and coinfections.

Frequency of Candidiasis and Trichomoniasis in different trimester among pregnant women were shown in Table 6.

\begin{tabular}{|c|c|c|}
\hline \multicolumn{3}{|c|}{$\begin{array}{c}\text { Table.1 Age wise distribution of patients having vaginal } \\
\text { discharge among pregnant women }\end{array}$} \\
\hline Age Group & No. Of patients & Percentage \\
\hline $\mathbf{2 0}-\mathbf{3 0}$ & 115 & 57.5 \\
\hline $\mathbf{3 0}-\mathbf{4 0}$ & 75 & 37.5 \\
\hline $\mathbf{4 0}-\mathbf{4 5}$ & 10 & 5 \\
\hline Total & $\mathbf{2 0 0}$ & $\mathbf{1 0 0}$ \\
\hline
\end{tabular}

\begin{tabular}{|l|l|l|l|l|l|}
\hline \multicolumn{5}{|c|}{ Table.2 Observation by Direct wet mount, Gram staining, Culture results of Candidiasis } \\
among pregnant women
\end{tabular}




\begin{tabular}{|c|c|c|c|c|c|c|}
\hline \multicolumn{7}{|c|}{$\begin{array}{l}\text { Table.3 Anti- fungal sensitivity pattern of the predominant Candida isolated from vaginal infection } \\
\text { among pregnant women }\end{array}$} \\
\hline Organisms & No. & $\begin{array}{l}\text { NS } \\
(\%)\end{array}$ & $\begin{array}{l}\text { FLC } \\
(\%)\end{array}$ & $\begin{array}{l}\text { AP } \\
(\%)\end{array}$ & $\begin{array}{l}\mathrm{CC} \\
(\%)\end{array}$ & $\begin{array}{l}\text { IT } \\
(\%)\end{array}$ \\
\hline Candida albicans & 36 & $26(72)$ & $31(86)$ & $32(88)$ & $33(91)$ & $28(77)$ \\
\hline $\begin{array}{l}\text { Candida } \\
\text { dublinansis }\end{array}$ & 3 & $02(66)$ & $03(100)$ & $02(66)$ & 03(100) & $03(100)$ \\
\hline Candida krusei & 2 & $02(100)$ & $01(50)$ & $02(100)$ & $02(100)$ & $02(100)$ \\
\hline Candida glabrata & 1 & 01(100) & $00(00)$ & $01(100)$ & 01(100) & $00(00)$ \\
\hline
\end{tabular}

\begin{tabular}{|c|l|l|l|}
\hline \multicolumn{3}{|c|}{ Table.4 Frequency of Trichomoniasis in various age groups among pregnant women } \\
\hline Age (yrs) & Total No of sample & $\begin{array}{l}\text { Total no. of positive } \\
\text { samples (\%) }\end{array}$ & $\begin{array}{l}\text { Positive no. of } \\
\text { Trichomoniasis (\%) }\end{array}$ \\
\hline $\mathbf{2 0 - 3 0}$ & 115 & $23(11.5)$ & $2(1)$ \\
\hline $\mathbf{3 0 - 4 0}$ & 75 & $18(9)$ & $1(0.5)$ \\
\hline $\mathbf{4 0 - 4 5}$ & 10 & $05(2.5)$ & $1(0.5)$ \\
\hline Total & $\mathbf{2 0 0}$ & $46(23)$ & $04(2)$ \\
\hline
\end{tabular}

\begin{tabular}{|l|l|r|c|c|c|}
\hline \multicolumn{5}{|c|}{ Table.5 Observation by Direct wet mount detection for motility, Giemsa, Acridine orange staining } \\
\hline of Trichomoniasis among pregnant women \\
\hline Organism & Samples & Motility & Giemsa stain & Acridine orange staining \\
\hline $\begin{array}{l}\text { Trichomoniasis } \\
\text { (N=04) }\end{array}$ & Positive & $\mathbf{4}$ & $\mathbf{2}$ & $\mathbf{3}$ \\
\hline & Negative & $\mathbf{4 2}$ & $\mathbf{2}$ & $\mathbf{1}$ \\
\hline
\end{tabular}

\begin{tabular}{|l|l|l|l|l|c|}
\hline \multicolumn{5}{|c|}{ Table.6 Frequency of Candidiasis and Trichomoniasis in different trimester among pregnant women } \\
\hline Trimester & No. of sample & Positive & Candidiasis & Trichomoniasis & $\begin{array}{l}\text { Co-infection } \\
\text { (Candida + Trichomonas })\end{array}$ \\
\hline $\mathbf{1}^{\text {st }}$ & 20 & $2(1 \%)$ & $2(1 \%)$ & $0(0 \%)$ & $0(0 \%)$ \\
\hline $\mathbf{2}^{\text {nd }}$ & 35 & $6(3 \%)$ & $5(2.5 \%)$ & $1(0.5 \%)$ & $1(0.5 \%)$ \\
\hline $\mathbf{3}^{\text {rd }}$ & 145 & $38(19 \%)$ & $35(17.5 \%)$ & $3(1.5 \%)$ & $3(1.5 \%)$ \\
\hline Total & 200 & $46(23 \%)$ & $42(21 \%)$ & $4(2 \%)$ & $4(2 \%)$ \\
\hline
\end{tabular}

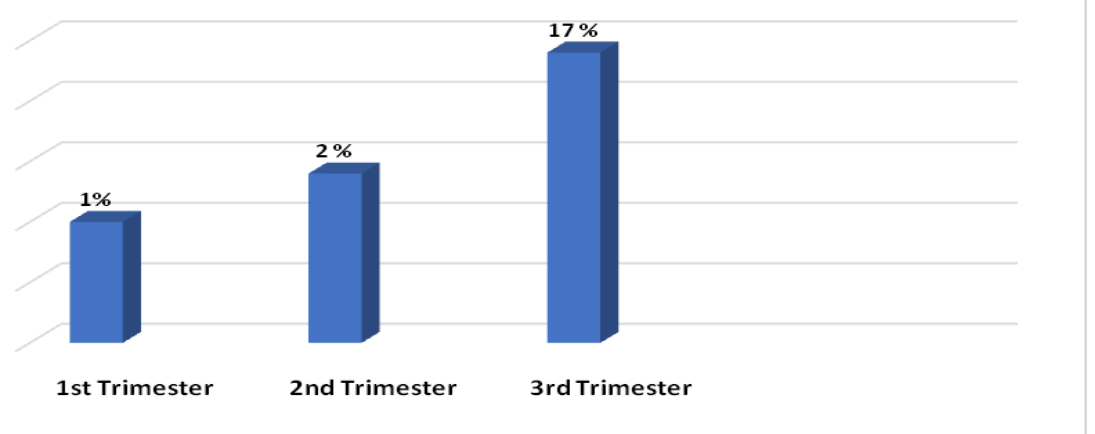

Figure 1: Percentage of Candidia sis in different trimester among pregnant women 


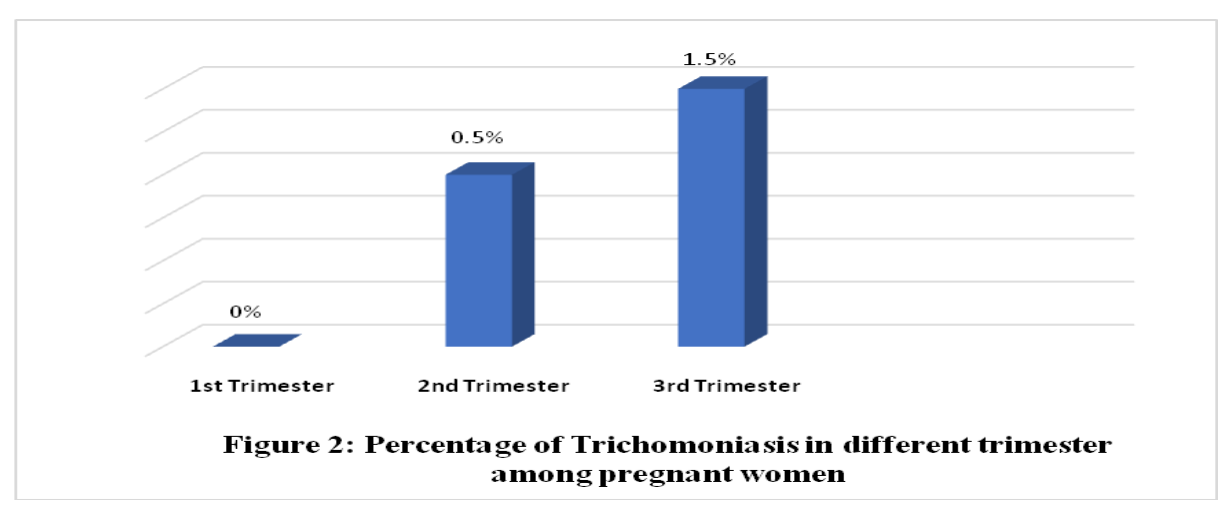

This study investigated, Co-infection of Candida albicans and Trichomonas vaginalis among pregnant woman attending the Tertiary Care Hospital. The present study observed and found prevalence of candidiasis was 21 $\%$, Trichomoniasis and co-infection $(\mathrm{T}+\mathrm{C})$ was $2 \%, 2 \%$ respectively.

\section{Vaginal discharge (VC)}

Vaginal discharge is a major problem in India among pregnant women. It occurs in $1-4 \%$ of all women with reproductive age group throughout the world and its prevalence in India was estimated approximate $30 \%$ by Thakur et al., 2010. ${ }^{14}$ The similar finding observed in our study, the prevalence of vaginal discharge was $23 \%$.

\section{Vulvovaginal candidiasis (VVC)}

Vulvovaginal candidiasis (VVC) is a common cause of vaginal discharge among pregnant women worldwide. ${ }^{15}$

The present study shows, high incidence (21\%) of vulvovaginal candidiasis among pregnant women. In similar study done by Parveen in $2008,{ }^{16}$ shows, incidence was $38 \%$ and Rekha and Jayoti, $2010^{17}$, reported 30\%, Sansui Mohhammed Ibrahim et al, ${ }^{18}$ also observed - 40\%, and Sobel et al, ${ }^{19}$ estimated that approximately $75 \%$ of women will experience an episode of VVC.

\section{Age wise distribution}

Vulvovaginitis is a common problem globally and affects woman of all age groups.

Present study observed the most common infective age group of $V V C$ and Tricomoniasis was 20-30 years than 30-40 years (Table 1).

Similar study done by Kemenbradikumo pondei et al., ${ }^{20}$ observed the most of VVC and $T$. vaginalis infection were in the age group of 21-30 years. In contrast study done by Alo M.N et al., ${ }^{21}$ shows; women within the age group of 31-35 years was high infection.

\section{Trimester wise distribution}

In the present study we observed, the infection of VVC was highest at third trimester 35(24\%) followed by second trimester $5(14 \%)$ then least was in the first trimester 2 (10\%) (Table 6). Similar finding reported by Alo et al., and Glatthaar et al., 21,22 , women within their 3ed trimester had the highest VVC infection (32\%)while those their $1^{\text {st }}$ trimester had least infection.

It has been observed that there is high concentration of oestrogen hormones during pregnancy and this provides favourable environment for the growth of candida, hence the highest prevalence in the third trimester. 


\section{Vulvovaginal triccomoniasis (VVT)}

In the present study, a low prevalence (2\%) was observed for trichomoniasis which is lower than the $10.6 \%$ reported by Mbu et al. ${ }^{23}$

Age \& trimester wise distribbution: Coinfection with Candida and Trichomonas vaginalis

The present study, out of 200 samples, 46 $(23 \%)$ cases of vaginal infection were positive among these women. of $4(2 \%)$ were coinfection infection.

Present study observed the most common infective age group of co-infection of Tricomoniasis \& Candidiasis was 20-30 years than 30-40 years (Table 6). In other studies done by Donbraye et al., 2010, Dickson Shey Nsagha et al., ${ }^{24}$ reported the higest coinfection reported within the age group of 2630 years and 26-40 years respectively. This could be as a result of the fact that this age group is still sexually active.

The women within their 3ed trimester had the highest co-infection $3(2 \%)$ while those their $2^{\text {nd }}$ trimester had least infection-1 $(2 \%)$. similar finding observed by Alo M.N (2012) et al., ${ }^{21,22}$. In contrast study done by Baekare et al., $2002^{23,}$ reported the co-infection in the $2^{\text {nd }}$ trimester also corroborates.

Many studies shows, $3^{\text {ed }}$ trimester are more vulnerable for vaginal infection then other two trimester, there may be repeated vaginal examination as well as pelvic examination in preparation for delivery.

In our study, most common vulvovaginal infection (VVI) is caused by vaginal Candidiasis $(21 \%)$ then trichomoniasis $(2 \%)$ among pregnant women. Similar Study done by Dr. Bhatia Ibrahim reported, vaginal
Candidiasis largely contributed to pathological vaginal discharge $37.1 \%$ while trichomoniasis contributed the least $(2.2 \%)^{26}$

In conclusion the pregnant women are more vulnerable to vaginal infection irrespective of their age, trimester and education level. Because of avoidance of contributory factors such as; douching, wearing tight underwear, general personal hygiene.

VVC, Trichomoniasis and other vaginal infection can resolve within a short period of time, if not treated timely then, it may enhances the growth of co-infection and it is a major cause of morbidity in pregnant women in developing countries like India. Hence there is need to create awareness education through campaign, symposium, seminar, conference and media on the detrimental effect of this dangerous co-infection like Candidiasis \& trichomoniasis among pregnant women. Rapid test should be mandatory routinely done for all pregnant women or in patients suffering from vaginal discharge and thus, help in early diagnosis and prompt treatment of patients.

This will ultimately prove beneficial to patients in reducing morbidity and associated adverse health outcomes in the form of pelvic inflammatory diseases and increased risk of acquiring other sexually transmitted diseases including human immunodeficiency virus infection.

\section{Acknowledgement}

We acknowledege and thanks to co-author, University \& Department for the suport.

\section{References}

1. Frobenius W, Bogdan C. Diagnostic value of vaginal discharge, wet mount and vaginal $\mathrm{pH}$ - an update on the basics 
of gynecologic infectiology. Geburtshilfe Frauenheilkd. 2015; 75(4): 355-66.

2. Workowski KA,Bolan GA.Sexually tramsmitted diseases treatment guidlines 2015 MMWR.Recommendation $\mathrm{s}$ and reports; Morbidity and Mortality weekly report.Recommandation and repotes / centers for disease control. Center for disease control and prevelance, 2015:1137.

3. Angebault C, Djossou F, Abélanet S, Permal E, Ben Soltana M, Diancourt L, et al., Candida albicans is not always the preferential yeast colonizing humans: a study in Wayampi Amerindians. $J$ Infect Dis. 2013; 208(10): 1705-16.

4. Weisseenbacher T.m. Witkin S.S gingelamaair A ET el Relasionship between recurrent valvovaginalcandidasis and immune mediators in vaginal fluid. Eur $\mathrm{j}$ of Obs \&Gyn and Rep Bio 144; 39.2009.6. Nyirijesy P. Chronic valvovaginal Candidiasis. Am FamaPhysician 63(4); 697-702.2001.

5. Cohen J: HIV Transmission - AIDS researchers look to Africa for new insights. Science 287, 942 (2000).

6. Fidel PL Jr: Host defense against oropharyngeal and Vaginal Candidiasis: site-specific differences. Rev Iberoam Micol 16(1), 8-15

7. El Ahmed HH, Cañadas-De la Fuente GA, Fernández-Castillo R, GonzálezJiménez E, Cantero-Hinojosa J,LardónFernández M: Generalized cutaneous Candidiasis in newborn at term. Biomedica 32(2), 170-173 (2012)9

8. Jatau ED, Olonitola OS, Olayinka AT: Prevalence of trichomonas infection among women attending antenatal clinics in Zaria, Nigeria. Annals Afr Med 5(4), 178-181 (2006)10

9. Mohanty S, Xess I, Hasan F, Kapil A,
Mittal S, Tolosa JE: Prevalence and susceptibility to fluconazole of Candida species causing Vulvovaginitis. Indian J Med Res 126(3), 216-219 (2007).

10. Khan, S.A, Amir, Ahaf. S and Tnveer, R. (2009). Evaluation of organisms causing vaginal discharge. J. Ayub. Abbotabad

11. Schwebke, J. R., and D. Burgess. 2004. Trichomoniasis. Clin. Microbiol. Rev. 17:794-803.

12. . Wiese, W., S. R. Patel, S. C. Patel, C. A. Ohl, and C. A. Estrada. 2000. A meta-analysis of the Papanicolaou smear and wet mount for the diagnosis of vaginal trichomoniasis. Am. J. Med. 108:301-308.

13. Schee CVD, Belkum AV, Zwijgers L, Brugge EVD, O’Neill EL, Luijendijk $\mathrm{AD}$, et al., Improved Diagnosis of Trichomonas vaginalis Infection by PCR Using Vaginal Swabs and Urine Specimens Compared to Diagnosis by Wet Mount Microscopy, Culture, and Fluorescent Staining. J Clin Microbiol. 1999; 37(12): 4127-30.

14. Thakur V.S. et al., 2010. Etiology and risk factors of recurrent vaginitis and its association with various contraceptives methods. IJMR,131: 83-87.

15. Agboola A, Vaginal discharge. In Text book of obstratrics \& gynae for medical students. $2^{\text {nd }}$ edition. edited by Ewawume EY, et al., 2005:72-79.

16. Parveen N, Munir AA, Din I, Majeed R. Frequency of vaginal candidiasis in pregnant women attending routine antenatal clinic, JCPSP (18): 154-157. 2008).

17. Rekha, S, Jayoti, S. 2010. Comparison of the visual, clinical and microbiological diagnosis of symptomatic vaginal discharge in the reproductive age group. Int.J.Pharm.Biomed.Res, 1:144-148)

18. Sansui Mohammed Ibrahim et al, 
management of abnormal vaginal discharge in pregnancy. Open access peeer- review chapter. 2016. 62599.

19. Sobel JD. Treatment of complicated Candida vaginitis, comparison of single and sequential dose of fluconazole. Am J Obstet Gynecol 2001;185:363)

20. Kemebradikumo pondei et al, the prevalence of symptomatic Vulvovaginal candidiasis and Trichomonas vaginalis infection and associated risk factors among woman in the Niger delta region of Nigeria JCPSP (18): 154157.2008.

21. Alo. M.N., Prevalence of asymptomatic co-infection of Candidiasis and vaginal trihomoniasis among pregnant woman in Abakaliki South-Eastern Nigeria J.N.S.R.2224-168).

22. Glatthaar et al., Vulvovaginitiscongresses. Vulvovaginitis-Round Table Discussion; Conference Proceedings, National Library of Australia; pp 37.

23. Barker A.A. Oni, A.A, Uma S.A.
Fayemiwo, N.A. et al., (2002) prevalence of Trichomanas vaginalis among commercial sex workers in Ibadam, Nigeria. ACEM4(2),25-29.

24. Mbu ER Kongnyuy EJ, Mbopi-keou Fx, Tonye, Nana et al., Gynaecological morbidity among HIV positive pregnant women in Cameroon, Reporod Health 2008; 3(9); 5:3.

25. Donbraye, E.O.O.B Okonkwo, IO. and Okandeji, IO-2010. Detection and prevalence of Trihomonas vaginalis among pregnant women in Ibadan, South Western Nigeria, WASJ. 11(12), 1512-1517.

26. Bhatia Ibrahim, et al., Prevalence and aetiology of pathological vaginal discharge among third trimester woman attending antenatal care at Kampala International University Teaching Hospital. IJHSR. Vol.7, issue: 11 Nov 2017.

\section{How to cite this article:}

Surbhi Mishra and Arti Jain. 2020. Co-infection of Candida albicans and Trichomonas vaginalis among Pregnant Women in Tertiary Care Hospital. Int.J.Curr.Microbiol.App.Sci. 9(09): 3061-3069. doi: https://doi.org/10.20546/ijcmas.2020.909.378 